\title{
Traumatic Brain Injury May Lead to Alzheimer's Disease and Related Dementia
}

\author{
Jian Shi* \\ Department of Neurology, Department of Veterans Affairs Medical Center, San Francisco and University of California, San Francisco, USA \\ *Corresponding author: Jian Shi, Department of Neurology, Department of Veterans Affairs Medical Center, San Francisco and \\ University of California, San Francisco, USA
}

\section{Introduction}

Traumatic brain injury (TBI) is a leading cause of death and disability in the US, particularly in those under age 40 , and $\sim 2 \%$ of the US population is living with a post-TBI associate syndrome and disorders, based on CDC reports. It is recently concerned that individuals living with TBI take an increased risk for developing several long-term health problems. An early study found that any history of brain injury increases the risk of developing Alzheimer's Disease (AD) and other dementia, and severe head trauma doubles the risk of developing $\mathrm{AD}$ dementia [1, 2]. Also, there is evidence that TBI may lower the age of onset of any dementia or AD [3], particularly in people with high rates of TBI, such as US and other veterans [4]. Today, it has been accepted that TBI may cause chronic traumatic encephalopathy (CTE), and some researchers have accepted that TBI as one of the AD risks may lead to AD development [5], but other researchers thought it is still exclusive [6]. In this review, we reviewed various pathological similarities between TBI and Alzheimer's Disease and Related Dementia (ADRD), which supports the view that TBI as one of AD risks may cause ADRD.

TBI may cause ADRD since its secondary injury mechanisms have several similarities with AD initiation. TBI results from an outward physical force that leads to immediate mechanical disturbance of brain tissue and follows by secondary injury events. Generally, secondary injuries occur in minutes to days, including oxidative stress, excitotoxin, calcium-influx, apoptosis, necrosis, hemorrhage, hypoxia, inflammation, etc. [7]. The principal pathologies seen in $\mathrm{AD}$ are amyloid beta $(\mathrm{A} \beta)$-contained plaques and neurofibrillary tangles (NFTs) containing hyper-phosphorylated tau (p-tau) protein. In $\mathrm{AD}$ development, $\mathrm{A} \beta$ is reported to trigger NMDAmediated $\mathrm{Ca}^{2+}$ influx, excitotoxicity; to exacerbate aging-related increases in oxidative stress; and to impair energy metabolism [8]; while TBI secondary injuries immediately cause excitotoxicity, $\mathrm{Ca}^{2+}$ influx, oxidative stress, etc. Moreover, oxidative stress alone can cause synapse disfunction and neuron death, leading to cognitive deficits [9], and oxidative stress can be seen in AD pathology via tau hyper-phosphorylation. Further, some primary kinases, including extracellular receptor kinase (ERK), calmodulin-dependent protein kinase (CaMKII), glycogen synthase kinase $3 \beta$ (GSK3 $\beta$ ) and cAMP response element-binding protein (CREB), are dynamically associated with oxidative stress-mediated abnormal hyper $\mathrm{p}$-tau. It suggests that alteration of these kinases could exclusively be involved in the pathogenesis of AD. Consistently, those primary kinases have also involved in the pathogenesis of TBI [10-12], although there are differences in the pathogenesis of TBI and AD.

Epidemiological studies have shown that TBI is a risk factor for tauopathies $[1,3,13]$, one of the two major pathological hallmarks of human AD. Usually, the tauopathies include tau hyperphosphorylation and aggregation. After a TBI event, p-tau and neurofibrillary tangles (NFTs) can be detected as early as $6 \mathrm{~h}$ $[14,15]$. While others examined p-tau expression in post-mortem brains many years after a TBI [16]. It has been found that NFTs levels elevated in approximately $30 \%$ individual's post-mortem who had a surviving moderate to severe $\mathrm{TBI}$, indicating the relationship between tau aggregation and a single TBI [16]. Sometimes, the pathological tau occurs in regions distant to the injury local that are synaptically connected, suggesting dissemination of tau aggregates [15]. Overall, TBI as a risk factor for tauopathies may induct both of tau hyperphosphorylation and aggregation. Most importantly, TBI has been suggested as a risk factor of AD from tauopathies by triggering disease onset and facilitating its progression, when tau deposition in areas vulnerable in aging and later mature areas in development [15]. 
Apolipoprotein E4 (ApoE4) is one of common genetic components between TBI and $\mathrm{AD}$ because it is closely related to neurogenesis-dysfunction and dementia. ApoE has three genotypes: 2, 3, and 4. Basically, ApoE4 is the most associated genetic risk factor for the development of AD and is expressed in more than half of the patients. However, it is estimated to be only $20 \%$ of the population. The presence of one or two ApoE4 alleles is increasing the AD risk by 3 or 12 folds, respectively [17], and also shifting the age of onset of dementia to a younger age [18]. Recent results suggest that ApoE4 is related to memory loss and overall cognitive dysfunction in patients with a history of mild TBI, but it does not affect people without neurotrauma [19].

Moreover, other researchers have proposed that ApoE4 alleles may be synergistic with TBI in increasing the risk for developing $\mathrm{AD}[20,21]$. Compared to other genotypes, ApoE4 is harmful in this process, as it inhibits neurite outgrowth, disturbs neuronal cytoskeleton, gathers amyloid $\beta$ protein $[22,23]$, and markedly aggravate tau-mediated degeneration [24]. Therefore, it is another important similarity between $\mathrm{TBI}$ and $\mathrm{AD}$, and a possible therapeutic target $[25,26]$.

Impaired adult hippocampal neurogenesis (AHN) were found in both TBI and AD animals [27, 28] and AD patients [29], which is one of the potential causes of dementia. AHN means that the additional new-born neurons are generated throughout life, and it is one of the unique phenomena of the adult mammalian brain and confers the plasticity of the entire hippocampus circuity. By studying the brains of $\mathrm{AD}$ patients, the number and maturation of these new-born neurons declined progressively with the progression of $\mathrm{AD}$, which provides evidence a potentially relevant mechanism underlying memory deficits in AD [29]. Consistent with this study, our previous study also showed that TBI impaired AHN, may lead to learning and memory deficits in rats [27].

Notably, ApoE is mainly expressed in astrocytes and secreted into the intercellular space that regulates other cells. It is also present in certain type I neural stem cells and neurons. Most importantly, ApoE is known to regulate postnatal neurogenesis in the hippocampus [30], whereas ApoE4 impairs AHN following TBI [31].

However, it is unclear that what are the relationships among TBI, AHN, ApoE4, and AD onset.

Given that TBI and ADRD involve many similarities, including secondary injury, tauopathies, ApoE4 and AHN, people after TBI may lead to ADRD and have long time healthy problems, especially those who TBI will cause tauopathies appearing in AD vulnerable areas. This may also give us more chances to study AD initiation and find novel therapeutic targets.

\section{References}

1. Sivanandam TM, Thakur MK (2012) Traumatic brain injury: a risk factor for Alzheimer's disease. Neurosci Biobehav Rev 36(5): 1376-1381.

2. Shively S, AI Scher, DP Perl, R Diaz-Arrastia (2012) Dementia resulting from traumatic brain injury: what is the pathology? Arch Neurol 69(10): 1245-1251.

3. Mendez MF (2017) What is the Relationship of Traumatic Brain Injury to Dementia? J Alzheimers Dis 57(3): 667-681.

4. Smith DH, VE Johnson, W Stewart (2013) Chronic neuropathologies of single and repetitive TBI: substrates of dementia? Nat Rev Neurol 9(4): 211-221.

5. Amakiri N, A Kubosumi, J Tran, PH Reddy (2019) Amyloid Beta and MicroRNAs in Alzheimer's Disease. Front Neurosci 13: Pp 430.

6. Julien J, S Joubert, MC Ferland, LC Frenette, MM Boudreau-Duhaime, et al. (2017) Association of traumatic brain injury and Alzheimer disease onset: A systematic review. Ann Phys Rehabil Med 60(5): 347-356.

7. D Laskowitz, G Grant (Editors), Boca Raton (FL) Krishnamurthy K, DT Laskowitz (2016) Cellular and Molecular Mechanisms of Secondary Neuronal Injury following Traumatic Brain Injury, in Translational Research in Traumatic Brain Injury.

8. Kamat PK, A Kalani, S Rai, S Swarnkar, S Tota, et al. (2016) Mechanism of Oxidative Stress and Synapse Dysfunction in the Pathogenesis of Alzheimer's Disease: Understanding the Therapeutics Strategies. Mol Neurobiol 53(1): 648-661.

9. Kamat PK, S Rai, S Swarnkar, R Shukla, S Ali, et al. (2013) Okadaic acid-induced Tau phosphorylation in rat brain: role of NMDA receptor Neuroscience 238: 97-113.

10. Shi J, DK Miles, BA Orr, SM Massa, SG Kernie (2007) Injury-induced neurogenesis in Bax-deficient mice: evidence for regulation by voltagegated potassium channels. Eur J Neurosci 25(12): 3499-512.

11. Farr SA, ML Niehoff, VB Kumar, DA Roby, JE Morley (2019) Inhibition of Glycogen Synthase Kinase 3beta as a Treatment for the Prevention of Cognitive Deficits after a Traumatic Brain Injury. J Neurotrauma 36(11): 1869-1875.

12. Rehman SU, M Ikram, N Ullah, SI Alam, HY Park, et al. (2019) Neurological Enhancement Effects of Melatonin against Brain InjuryInduced Oxidative Stress, Neuroinflammation, and Neurodegeneration via AMPK/CREB Signaling. Cells 8(7): 760

13. Gilbert M, C Snyder, C Corcoran, MC Norton, CG Lyketsos, et al. (2014) The association of traumatic brain injury with rate of progression of cognitive and functional impairment in a population-based cohort of Alzheimer's disease: the Cache County Dementia Progression Study. Int Psychogeriatr 26(10): 1593-1601.

14. Grady MS, MR McLaughlin, CW Christman, AB Valadka, CL Fligner, et al. (1993) The use of antibodies targeted against the neurofilament subunits for the detection of diffuse axonal injury in humans. J Neuropathol Exp Neurol 52(2): 143-152.

15. Edwards G 3rd, J Zhao, PK Dash, C Soto, I Moreno Gonzalez (2019) Traumatic Brain Injury Induces Tau Aggregation and Spreading. J Neurotrauma 37(1): 80-92.

16. Johnson VE, W Stewart, DH Smith (2012) Widespread tau and amyloidbeta pathology many years after a single traumatic brain injury in humans. Brain Pathol 22(2): 142-149.

17. Yu JT, L Tan, J Hardy (2014) Apolipoprotein E in Alzheimer's disease: an update. Annu Rev Neurosci 37: 79-100.

18. Corder EH, AM Saunders, W Strittmatter, DE Schmechel, PC Gaskell, et al. (1993) Gene dose of apolipoprotein E type 4 allele and the risk of Alzheimer's disease in late onset families. Science 261(5123): 921-923. 
19. Merritt VC, KM Lapira, AL Clark, SF Sorg, ML Werhane, et al. (2018) APOE-epsilon4 Genotype is Associated with Elevated Post-Concussion Symptoms in Military Veterans with a Remote History of Mild Traumatic Brain Injury. Arch Clin Neuropsychol 34(5): 706-712.

20. Mayeux R, R Ottman, G Maestre, C Ngai, MX Tang, et al. (1995) Synergistic effects of traumatic head injury and apolipoprotein-epsilon 4 in patients with Alzheimer's disease. Neurology 45(3 Pt 1): 555-557.

21. Katzman R, DR Galasko, T Saitoh, X Chen, MM Pay, et al. (1996) Apolipoprotein-epsilon 4 and head trauma: Synergistic or additive risks? Neurology 46(3): 889-891

22. Mahley RW, KH Weisgraber, Y Huang (2006) Apolipoprotein E4: a causative factor and therapeutic target in neuropathology, including Alzheimer's disease. Proc Natl Acad Sci U S A 103(15): 5644-5651.

23. Ji ZS, RD Miranda, YM Newhouse, KH Weisgraber, Y Huang, et al. (2002) Apolipoprotein E4 potentiates amyloid beta peptide-induced lysosomal leakage and apoptosis in neuronal cells. J Biol Chem 277(24): 2182121828.

24. Shi Y, K Yamada, SA Liddelow, ST Smith, L Zhao, et al. (2017) ApoE4 markedly exacerbates tau-mediated neurodegeneration in a mouse model of tauopathy. Nature 549(7673): 523-527.

25. Safieh M, AD Korczyn, DM Michaelson (2019) ApoE4: an emerging therapeutic target for Alzheimer's disease. BMC Med 17(1): 64.
26. Belloy ME, V Napolioni, MD Greicius (2019) A Quarter Century of APOE and Alzheimer's Disease: Progress to Date and the Path Forward. Neuron 101(5): 820-838.

27. Shi J, FM Longo, SM Massa (2013) A small molecule p75(NTR) ligand protects neurogenesis after traumatic brain injury. Stem Cells 31(11): 2561-2574.

28. Chavoshinezhad S, HM Kouchesfahani, A Ahmadiani, L Dargahi (2019) Interferon beta ameliorates cognitive dysfunction in a rat model of Alzheimer's disease: Modulation of hippocampal neurogenesis and apoptosis as underlying mechanism. Prog Neuropsychopharmacol Biol Psychiatry 94: 109661.

29. Moreno Jimenez EP, M Flor Garcia, J Terreros Roncal, A Rabano, F Cafini, et al. (2019) Adult hippocampal neurogenesis is abundant in neurologically healthy subjects and drops sharply in patients with Alzheimer's disease. Nat Med 25(4): 554-560.

30. Tensaouti Y, EP Stephanz, TS Yu, SG Kernie (2018) ApoE Regulates the Development of Adult Newborn Hippocampal Neurons. eNeuro 5(4): ENEUR0.0155-18.

31.Hong S, PM Washington, A Kim, CP Yang, TS Yu, et al. (2016) Apolipoprotein E Regulates Injury-Induced Activation of Hippocampal Neural Stem and Progenitor Cells. J Neurotrauma 33(4): 362-374.

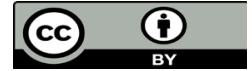

This work is licensed under Creative Commons Attribution 4.0 License

To Submit Your Article Click Here: Submit Article

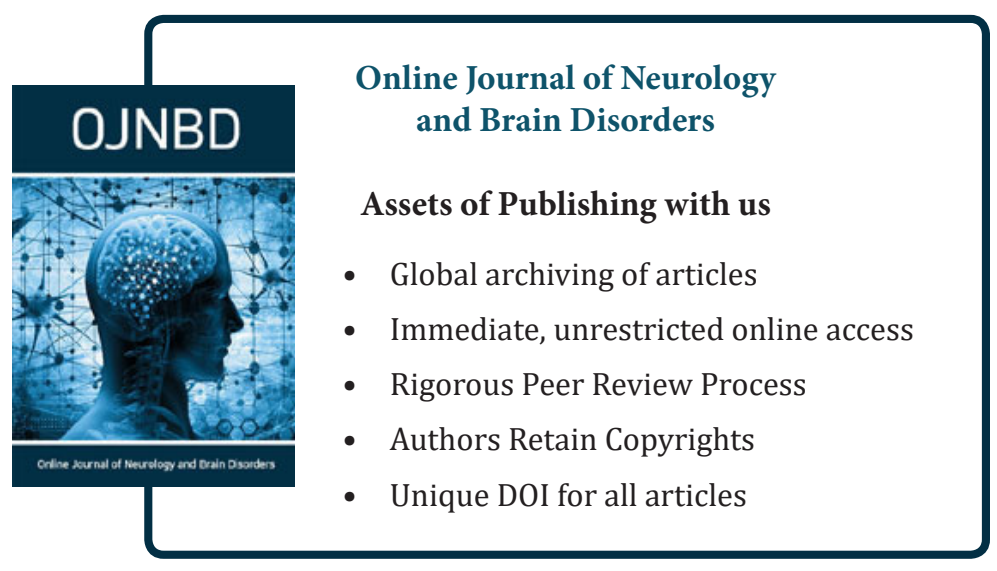

